\title{
Valuation theory, generalized IFS attractors and fractals
}

\author{
JAn Dobrowolski And Franz-ViKTor Kuhlmann
}

\begin{abstract}
Using valuation rings and valued fields as examples, we discuss in which ways the notions of "topological IFS attractor" and "fractal space" can be generalized to cover more general settings.
\end{abstract}

Mathematics Subject Classification. Primary 28A80, 12J25; Secondary $37 \mathrm{C} 25,37 \mathrm{C} 70,12 \mathrm{~J} 15$.

Keywords. Fractals, Iterated function systems, Valued fields.

Given functions $f_{1}, \ldots, f_{n}$ on a set $X$, we will associate to them an iterated function system (IFS), denoted by

$$
F=\left[f_{1}, \ldots, f_{n}\right]
$$

where we view $F$ as a function on the power set $\mathcal{P}(X)$ defined by

$$
\mathcal{P}(X) \ni S \mapsto F(S):=\bigcup_{i=1}^{n} f_{i}(S) .
$$

One of the basic approaches to calling a space $X$ "fractal" is to ask that there is an iterated function system $F$ such that $F(X)=X$, and that the functions in the system satisfy certain additional forms of being "contracting":

Definition 1. A compact metric space $(X, d)$ is called fractal if there is an iterated function system $F=\left[f_{1}, \ldots, f_{n}\right]$ with $F(X)=X$ where the functions $f_{i}$ are weakly contracting, that is, $d\left(f_{i} x, f_{i} y\right)<d(x, y)$ for any distinct $x, y \in$ $X$.

The first author was supported by European Union's Horizon 2020 research and innovation programme under the Marie Skłodowska-Curie Grant Agreement No. 705410. The authors wish to thank the referee for his corrections and suggestions that helped to improve this paper. 
Alternatively, one may ask that the functions $f_{i}$ are contracting, that is, there is some positive real number $C<1$ such that $d\left(f_{i} x, f_{i} y\right) \leq C d(x, y)$ for all $x, y \in X$.

Iterated function systems consisting of weakly contracting functions are studied in, e.g., $[4,5,7]$.

In the absence of a metric, one has to find other ways of encoding what is meant by "contracting". In [1], Banakh and Nowak give a topological analogue for the common definition of "fractal" that uses iterated function systems; for a detailed continuation of this approach, see $[2,3]$.

Definition 2. A compact topological space $X$ is called fractal if there is an iterated function system $F=\left[f_{1}, \ldots, f_{n}\right]$ consisting of continuous functions $f_{i}: X \rightarrow X$ such that $F(X)=X$ and the following "shrinking condition" is satisfied:

(SC) for every open covering $\mathcal{C}$ of $X$, there is some $k \in \mathbb{N}$ such that for every sequence $\left(i_{1}, \ldots, i_{k}\right) \in\{1, \ldots, n\}^{k}$ there is $U \in \mathcal{C}$ with

$$
f_{i_{1}} \circ \cdots \circ f_{i_{k}}(X) \subset U
$$

Clearly, it suffices to check (SC) only for finite coverings. If we fix a basis for the open sets, then it suffices to check (SC) only for finite coverings consisting of basic sets, as every finite covering can be refined to such a covering.

We will now give examples of iterated function systems in the special case where the topology is induced by a valuation on a field. For general background on valuation theory, see, e.g., [6,8-10]. For the topology induced by a valuation $v$ on a field $K$ with value group $v K$, one can take the collection of ultrametric balls

$$
B_{\alpha}(a):=\{b \in K \mid v(a-b) \geq \alpha\} \text { where } \alpha \in v K \text { and } a \in K
$$

as a basis; note that by the ultrametric triangle law, this set is closed under nonempty intersections over finite subsets. For the same reason, if $v(a-b) \geq \alpha$, then $B_{\alpha}(a)=B_{\alpha}(b)$. The same works when we restrict $v$ to a subring $R$ of $K$, except that then the values of the elements in $R$ just form a linearly ordered subset of $v K$.

Example 3. We take a prime $p$ and denote by $\mathbb{F}_{p}$ the finite field with $p$ elements. Then $\mathbb{F}_{p}$ consists of the elements $\bar{i}=i+p \mathbb{Z}, 0 \leq i<p$. We consider the Laurent series ring

$$
R:=\mathbb{F}_{p}[[t]]=\left\{\sum_{j=0}^{\infty} \bar{i}_{j} t^{j} \mid \bar{i}_{j} \in \mathbb{F}_{p}\right\} .
$$

The $t$-adic valuation $v_{t}$ on $\mathbb{F}_{p}[[t]]$ is defined by

$$
v_{t} \sum_{j=0}^{\infty} \overline{i_{j}} t^{j}=\min \left\{j \mid j \geq 0 \text { such that } \overline{i_{j}} \neq \overline{0}\right\} \text {. }
$$


For $0 \leq i \leq p-1$, we define a function $f_{i}$ by

$$
f_{i}\left(\sum_{j=0}^{\infty} \bar{i}_{j} t^{j}\right):=\bar{i}+\sum_{j=1}^{\infty} \bar{i}_{j-1} t^{j}=\bar{i}+t \sum_{j=0}^{\infty} \overline{i_{j}} t^{j}
$$

where $\bar{i}$ is understood to be an element of $\mathbb{F}_{p}$. Then

$$
f_{i}(R)=\bar{i}+t R
$$

and therefore, the iterated function system $F=\left[f_{0}, \ldots, f_{p-1}\right]$ satisfies $F(R)=$ $\bigcup_{0 \leq i<p} \bar{i}+t R=R$. Each ultrametric ball in $R$ (with respect to the $t$-adic valuation of $\left.\mathbb{F}_{p}[[t]]\right)$ is of the form

$$
B_{m}\left(\sum_{j=0}^{m-1} \overline{i_{j}} t^{j}\right)=\left\{b \in R \mid t^{m} \text { divides } b-\sum_{j=0}^{m-1} \overline{i_{j}} t^{j}\right\}
$$

for some integer $m \geq 0$, which we call the radius of the ball. (The empty sum is understood to be 0 ). Given any finite open covering of $R$ consisting of ultrametric balls, we take $m$ to be the maximum of the radii of all balls in the covering. Then the covering can be refined to a covering of the form

$$
\left\{B_{m}\left(\sum_{j=0}^{m-1} \overline{i_{j}} t^{j}\right) \mid i_{0}, \ldots, i_{m-1} \in\{0, \ldots, p-1\}\right\} .
$$

Choose any $m \geq 1$ and $i_{0}, \ldots, i_{m-1} \in\{0, \ldots, p-1\}$. By induction on $m$ we derive from (2):

$$
f_{i_{0}} \circ \cdots \circ f_{i_{m-1}}(R)=\overline{i_{0}}+\overline{i_{1}} t+\cdots+\overline{i_{m-1}} t^{m-1}+t^{m} R=B_{m}\left(\sum_{j=0}^{m-1} \overline{i_{j}} t^{j}\right) .
$$

Since the functions $f_{i}$ are continuous in the topology induced by the ultrametric (an argument will be given below in the more general case of discrete valuation rings), we see that $\mathbb{F}_{p}[[t]]$ with its ultrametric balls is fractal, in the sense of Definition 2.

Here is an obvious generalization of the previous example.

Example 4. We work in the same situation as in the last example, but now fix an integer $\mu \geq 0$ and for every $i^{*}=\left(i^{*}(0), \ldots, i^{*}(\mu)\right) \in\{0, \ldots, p-1\}^{\mu+1}$ we set

$$
f_{i^{*}}\left(\sum_{j=0}^{\infty} \overline{i_{j}} t^{j}\right):=\sum_{j=0}^{\mu} \overline{i^{*}(j)} t^{j}+t^{\mu+1} \sum_{j=0}^{\infty} \overline{i_{j}} t^{j}
$$

Then

$$
f_{i^{*}}(R)=\sum_{j=0}^{\mu} \overline{i^{*}(j)} t^{j}+t^{\mu+1} R
$$


and therefore, the iterated function system $F=\left[f_{i^{*}} \mid i^{*} \in\{0, \ldots, p-1\}^{\mu+1}\right]$ satisfies

$$
F(R)=\sum_{j=0}^{\mu} \mathbb{F}_{p} t^{j}+t^{\mu+1} R=R
$$

Choose any $m \geq 1$ and $i_{0}^{*}, \ldots, i_{m-1}^{*} \in\{0, \ldots, p-1\}^{\mu+1}$. By induction on $m$ we derive from $(3)$ :

$$
\begin{aligned}
f_{i_{0}^{*}} \circ \cdots \circ f_{i_{m-1}^{*}}(R)= & \sum_{j=0}^{\mu} \overline{i_{0}^{*}(j)} t^{j}+t^{\mu+1} \sum_{j=0}^{\mu} \overline{i_{1}^{*}(j)} t^{j}+\cdots \\
& +\left(t^{\mu+1}\right)^{m-1} \sum_{j=0}^{\mu} \overline{i_{m-1}^{*}(j)} t^{j}+\left(t^{\mu+1}\right)^{m} R \\
= & B_{m(\mu+1)}\left(\sum_{k=0}^{m-1} t^{k(\mu+1)} \sum_{j=0}^{\mu} \overline{i_{k}^{*}(j)} t^{j}\right) .
\end{aligned}
$$

We generalize our observations to discrete valuation rings (which in general cannot be presented in power series form, in particular not in mixed characteristic).

We take a discrete valuation ring $R$ with maximal ideal $M$ and choose a uniformizing parameter $t \in R$, i.e., the value of $t$ is the smallest positive element in the value set of $R$. Further, we choose a system of representatives $S \subset R$ for the residue field $R / M$. Then for every $s \in S$ we define a function $f_{s}$ by:

$$
f_{s}(a):=s+t a
$$

for $a \in R$. Then

$$
f_{s}(R)=s+t R
$$

and therefore,

$$
\bigcup_{s \in S} f_{s}(R)=\bigcup_{s \in S} s+t R=R
$$

Choose any $m \geq 1$ and $s_{0}, \ldots, s_{m-1} \in S$. By induction on $m$ we derive from (5):

$f_{s_{0}} \circ \cdots \circ f_{s_{m-1}}(R)=s_{0}+s_{1} t+\cdots+s_{m-1} t^{m-1}+t^{m} R=B_{m}\left(\sum_{j=0}^{m-1} s_{j} t^{j}\right)$.

If $a, b \in R$ with $a-b \in B_{m}(0)=t^{m} R$, then

$$
f_{s}(a)-f_{s}(b)=s+t a-(s+t b)=t(a-b) \in t^{m+1} R=B_{m+1}(0) .
$$

This shows that each $f_{s}$ is contracting and hence continuous in the topology induced by the ultrametric.

If $R / M$ is finite, then we have finitely many functions and obtain: 
Proposition 5. Every discrete valuation ring with finite residue field and equipped with the canonical ultrametric is fractal (under both definitions given above).

Note that for any topological space $X$, the existence of a continuous IFS $F=\left[f_{1}, \ldots, f_{n}\right]$ satisfying conditions $(S C)$ and $X=\bigcup_{i} f_{i}[X]$ from Definition 2 implies that $X$ is quasi-compact. By the following example, it can be seen that these conditions do not imply that $X$ is Hausdorff (so Definition 2 could be also considered for non-Hausdorff quasicompact spaces):

Example 6. Let $X=[0,1]$ be equipped with the topology in which the open sets are $\emptyset$ and the cofinite sets. Define $f_{1}, f_{2}: X \rightarrow X$ by $f_{1}(x)=x / 2$, $f_{2}(x)=1 / 2+x / 2$. Then the system $\left(f_{1}, f_{2}\right)$ consists of continuous functions, and satisfies conditions $(S C)$ and $X=\bigcup_{i} f_{i}[X]$.

The following definition seems to be the weakest reasonable generalization of Definition 2 to possibly infinite function systems.

Definition 7. Let $X$ be a topological space, and $\left\{f_{i}: i \in I\right\}$ any set of continuous mappings $X \rightarrow X$ satisfying $(S C)$, i.e., for any finite open covering $\mathcal{U}$ of $X$ there is a natural number $l$ such that for any $g_{1}, \ldots, g_{l} \in\left\{f_{i}: i \in I\right\}$, the image $g_{1} \circ \cdots \circ g_{l}[X]$ is contained in some $U \in \mathcal{U}$. We will say that $X$ is a topological attractor for $\left\{f_{i}: i \in I\right\}$ if $X=\operatorname{cl}\left(\bigcup_{i \in I} f_{i}[X]\right)$. For any cardinal number $\kappa$, we will say that $X$ is a topological $\kappa$-IFS-attractor if $X$ is an attractor for some set of continuous functions satisfying $(S C)$ of cardinality at most $\kappa$.

This definition generalizes Definition 2 in the sense that being a topological IFS-attractor is the same as being a compact $n$-IFS-attractor for some $n \in \omega$ (because when $X$ is compact and $I$ is finite, then we have $\operatorname{cl}\left(\bigcup_{i \in I} f_{i}[X]\right)=$ $\left.\bigcup_{i \in I} f_{i}[X]\right)$.

The reader may note that our notion of a topological attractor is not the same as the one used in papers [5,7], where a nonempty compact set is defined to be a topological attractor if it is homeomorphic to an attractor.

For normal spaces, the property of being a $\kappa$-IFS-attractor implies a bound on the weight (i.e., the minimal cardinality of a basis of the topology):

Proposition 8. Suppose $X$ is a normal space which is a $\kappa$-IFS-attractor. Then $w(X) \leq 2^{\kappa}+\aleph_{0}$.

Proof. Choose a system of functions $F=\left\{f_{i}: i \in I\right\}$ of cardinality at most $\kappa$ satisfying $(S C)$ such that $X$ is an attractor for $F$, i.e., $X=\operatorname{cl}\left(\bigcup_{i \in I} f_{i}[X]\right)$.

Claim 1. For any natural number $l$, we have that

$$
X=\mathrm{cl}\left(\bigcup_{g_{1}, \ldots, g_{l} \in F} g_{1} \circ \cdots \circ g_{l}[X]\right)
$$


Proof of the claim. We proceed by induction on $l$. Suppose that (6) holds. Then for every $i \in I$, we get by the continuity of $f_{i}$ that

$$
\begin{aligned}
f_{i}[X] & \subset \operatorname{cl}\left(f_{i}\left[\bigcup_{g_{1}, \ldots, g_{l} \in F} g_{1} \circ \cdots \circ g_{l}[X]\right)\right. \\
& =\operatorname{cl}\left(\bigcup_{g_{1}, \ldots, g_{l} \in F} f_{i} \circ g_{1} \circ \cdots \circ g_{l}[X]\right) \\
& \subset \operatorname{cl}\left(\bigcup_{g_{1}, \ldots, g_{l+1} \in F} g_{1} \circ \cdots \circ g_{l+1}[X]\right) .
\end{aligned}
$$

Thus, we obtain that $X=\operatorname{cl}\left(\bigcup_{i \in I} f_{i}[X]\right) \subset \operatorname{cl}\left(\bigcup_{g_{1}, \ldots, g_{l+1} \in F} g_{1} \circ \cdots \circ g_{l+1}[X]\right)$. This completes the proof of the claim.

Define

$$
\mathcal{B}=\left\{X \backslash \mathrm{cl}\left(\bigcup_{\left(g_{1}, \ldots, g_{l}\right) \in I} g_{1} \circ \cdots \circ g_{l}[X]\right) \mid l<\omega, I \subset F^{l}\right\} .
$$

Clearly, $|\mathcal{B}| \leq \sum_{l<\omega}\left|P\left(F^{l}\right)\right|=2^{\kappa} \aleph_{0}=2^{\kappa}+\aleph_{0}$. We will show that $\mathcal{B}$ is a basis of $X$. So take any open subset $U$ of $X$ and $x \in U$. Since $X$ is normal, we can choose open sets $V_{1}, V_{2}$ such that

$$
x \in V_{1} \subset \operatorname{cl}\left(V_{1}\right) \subset V_{2} \subset \operatorname{cl}\left(V_{2}\right) \subset U .
$$

Let $l$ be as in the condition $(S C)$ for $F$ and the covering $\left\{V_{2}, X \backslash \operatorname{cl}\left(V_{1}\right)\right\}$ of $X$. Define $J=\left\{\left(g_{1}, \ldots, g_{l}\right) \in F^{l}: g_{1} \circ \cdots \circ g_{l}[X] \subset X \backslash \operatorname{cl}\left(V_{1}\right)\right\}$ and $W=$ $X \backslash \operatorname{cl}\left(\bigcup_{\left(g_{1}, \ldots, g_{l}\right) \in J} g_{1} \circ \cdots \circ g_{l}[X]\right) \in \mathcal{B}$. Since $V_{1}$ is disjoint from $\bigcup_{\left(g_{1}, \ldots, g_{l}\right) \in J} g_{1} \circ$ $\cdots \circ g_{l}[X]$, we get that $x \in W$. It remains to check that $W \subset U$. Take any $y \in X \backslash U$. We will show that $y \in \operatorname{cl}\left(\bigcup_{\left(g_{1}, \ldots, g_{l}\right) \in J} g_{1} \circ \cdots \circ g_{l}[X]\right)$. Take any open neighbourhood $Z$ of $y$. By the claim, $g_{1} \circ \cdots \circ g_{l}[X]$ meets $Z \cap\left(X \backslash \operatorname{cl}\left(V_{2}\right)\right)$ for some $h_{1}, \ldots, h_{l} \in F$. Then the image $g_{1} \circ \cdots \circ g_{l}[X]$ is not contained in $V_{2}$, so it is contained in $X \backslash \operatorname{cl}\left(V_{1}\right)$ and $\left(h_{1}, \ldots, h_{l}\right) \in J$. Therefore, $Z$ meets $\bigcup_{\left(g_{1}, \ldots, g_{l}\right) \in J} g_{1} \circ \cdots \circ g_{l}[X]$, and we are done.

The above proposition applies in particular to compact spaces (which are known to be normal). In particular, we obtain that every topological IFSattractor has a countable basis. Thus, by the Urysohn metrization theorem, we get:

Corollary 9. Every topological IFS-attractor is metrizable.

Condition $(S C)$ is not satisfied in some natural examples where the metric shrinking condition is satisfied (i.e., $\lim _{l} \sup _{i_{1}, \ldots, i_{l}} \operatorname{diam}\left(f_{i_{1}} \circ \cdots \circ f_{i_{l}}[X]\right)=0$ ):

Example 10. Let $X=\omega^{\omega}$ be the Baire space (which is homeomorphic to $k((t)$ ) considered with the valuation topology, where $k$ is any field of cardinality $\left.\aleph_{0}\right)$. For any $i<\omega$, define $f_{i}: X \rightarrow X$ as follows: $f_{i}(x)(0)=i$ and $f_{i}(x)(n)=$ $x(n-1)$ for $n>0$. Then $(S C)$ is not satisfied for $f_{i}, i<\omega$, which is witnessed 
by the covering $\{U, X \backslash U\}$, where $U=\bigcup_{n<\omega}\{x \in X: x(0)=n, x(1)=\cdots=$ $x(n)=0\}$.

Thus, we want to consider another topological shrinking condition, in which we are allowed to choose a basis from which the covering sets are taken. However, to make it possible to cover in this way the whole space (which is not assumed to be compact), we allow one of the covering sets to be not in the fixed basis. This leads to the following definition:

Definition 11. A family of functions $\left(f_{i}\right)_{i \in I}$ on a topological space $X$ satisfies $(S C *)$ if there is a basis $\mathcal{B}$ of $X$ such that for every finite open covering $\mathcal{C}$ of $X$ containing at most one set which is not in $\mathcal{B}$, there is some $k \in \mathbb{N}$ such that, for every sequence $\left(i_{1}, \ldots, i_{k}\right) \in I^{k}$, there is $U \in \mathcal{C}$ with

$$
f_{i_{1}} \circ \cdots \circ f_{i_{k}}(X) \subset U \text {. }
$$

Every space is an attractor for the set of all constant functions from $X$ to $X$ (i.e., is covered by their images). We will say that $X$ is a weak $*$-IFS attractor if it is an attractor for a set of functions satisfying $(S C *)$ of a cardinality smaller than $|X|$. We will say that $X$ is a $*$-IFS attractor if it is an attractor for a finite set of functions satisfying $(S C *)$.

Clearly, we have:

Remark 12. If $X$ is a compact space, then it is a $*$-IFS attractor if and only if it is a topological IFS attractor.

By the following example, it can be seen that being a $*$-IFS attractor does not imply compactness:

Example 13. Let $X=\omega$ be considered with the discrete topology. Define $f_{0}, f_{1}: X \rightarrow X$ by $f_{0}(n)=0$ and $f_{1}(n)=n+1$. Then $X$ is a $*$-IFS attractor for $\left\{f_{0}, f_{1}\right\}$, so $X$ is a $*$-IFS attractor.

Proof. We choose a basis $\mathcal{B}$ consisting of all singletons. Consider any covering of $X$ of a form $\left\{U,\left\{n_{1}\right\}, \ldots,\left\{n_{l}\right\}\right\}$. Then it is sufficient to take $k=$ $\max \left(n_{1}, \ldots, n_{l}\right)+1$.

Example 14. Let $X=\omega^{\omega}$, and let $f_{i}: X \rightarrow X, i<\omega$ be as in Example 10 . Then, $\left(f_{i}\right)_{i<\omega}$ satisfies $(S C *)$, so $X$ is a weak $*$-IFS attractor. More generally, for any cardinal number $\kappa$, the space $\kappa^{\omega}$ is an attractor for a set of functions of cardinality $\kappa$, so it is a weak $*$-IFS attractor if $\kappa<\kappa^{\omega}$ (this holds for example for all cardinals with countable cofinality, so for unboundedly many cardinals).

Proof. For any $\alpha \in \kappa$, define $f_{\alpha}: \kappa^{\omega} \rightarrow \kappa^{\omega}$ by $f_{\alpha}(x)(0)=\alpha$ and $f_{\alpha}(x)(n)=$ $x(n-1)$ for $n>0$. We choose the standard basis of $\kappa^{\omega}$, i.e.,

$$
\mathcal{B}=\left\{A_{x}: x \in \kappa^{k}, k<\omega\right\},
$$

where $A_{x}=\left\{y \in \kappa^{\omega}: x \subset y\right\}$. Write $|x|=k$ if $x \in \kappa^{k}$. Choose any open covering of $\kappa^{\omega}$ of the form $\left\{U, A_{x_{1}}, \ldots, A_{x_{n}}\right\}$. Put $k=\max \left(\left|x_{1}\right|, \ldots,\left|x_{n}\right|\right)$. For any sequence $\left(\alpha_{0}, \ldots, \alpha_{k-1}\right) \in \kappa^{k}$ we have that $f_{\alpha_{0}} \circ \cdots \circ f_{\alpha_{k-1}}\left[\kappa^{\omega}\right]=A_{y}$, where $y(i)=\alpha_{i}$ for all $i<k$, so this image is either contained in one of the sets $A_{x_{1}}, \ldots, A_{x_{n}}$ or disjoint from all of them and thus contained in $U$. 
Proposition 15. Suppose $A$ is a densely ordered abelian group and $|\cdot|: A \rightarrow$ $\{a \in A \mid a \geq 0\}$ is the associated absolute value. Consider a collection of functions $f_{i}: A \rightarrow A, i \in I$. Suppose that there is a sequence $\left(a_{i}\right)_{i<\omega}$ of positive elements of $A$ which converges to 0 , and that for every $k$ and any sequence $\left(i_{1}, \ldots, i_{k}\right) \in I^{k}$ we have $\operatorname{diam}\left(f_{i_{1}} \circ \cdots \circ f_{i_{k}}[A]\right)<a_{i}$. Then $f_{i}: A \rightarrow A, i \in I$ satisfies $S C *$ (where we consider $A$ with the order topology).

Proof. We choose a basis $\mathcal{B}$ of the order topology on $A$ consisting of all open intervals. We consider any covering of $A$ of the form $\left\{U,\left(a_{1}, b_{1}\right), \ldots,\left(a_{n}, b_{n}\right)\right\}$. For any $i$ there is $c_{i}>0$ such that each of the intervals $\left(a_{i}-c_{i}, a_{i}+c_{i}\right)$, $\left(b_{i}-c_{i}, b_{i}+c_{i}\right)$ is contained in one of the sets from the covering. Now, choose $k$ such that for every sequence $\left(i_{1}, \ldots, i_{k}\right) \in I^{k}$ we have $\operatorname{diam}\left(f_{i_{1}} \circ \cdots \circ f_{i_{k}}[A]\right)<$ $c:=1 / 2 \min \left(c_{1}, \ldots, c_{n}, b_{1}-a_{1}, \ldots, b_{n}-a_{n}\right)$. Then for $a \in A,[a-c, a+c]$ is a subset of some of the sets from the covering: otherwise, by the choice of $c_{i}$ 's, $a$ would be at distance $>c$ from all $a_{i}$ 's and $b_{i}$ 's, and hence $a$ could not belong to any of the intervals $\left(a_{i}, b_{i}\right)$ (as in that case we would have $[a-c, a+c] \subset\left(a_{i}, b_{i}\right)$ ). But that would mean that $[a-c, a+c] \subset U$.

By the above, any set of diameter smaller than $c$ is contained in one of the sets from the covering, so we are done.

Corollary 16. $\mathbb{R}$ is a weak $*-I F S$ attractor.

Proof. Take a continuous bijection $f_{0}: \mathbb{R} \rightarrow(-1,1)$ which is Lipschitz with constant $1 / 2$. Define $f_{n}(x)=n+f_{0}(x)$ for any integer $n$. Then, clearly, the family $\left\{f_{n}: n \in \mathbb{Z}\right\}$ satisfies the assumptions of Proposition 15, so we obtain that it satisfies $S C *$. Of course, $\mathbb{R}$ is an attractor for that family (and has a bigger cardinality).

A fractal space is compact. If we have a space that is only locally compact, one can ask whether it is "locally fractal", that is, whether every element is contained in a fractal subspace.

Example 17. We consider the Laurent series field

$$
K:=\mathbb{F}_{p}((t))=\left\{\sum_{j=\ell}^{\infty} \overline{i_{j}} t^{j} \mid \ell \in \mathbb{Z}, \overline{i_{j}} \in \mathbb{F}_{p}\right\} .
$$

The $t$-adic valuation $v_{t}$ on $\mathbb{F}_{p}((t))$ is defined by

$$
v_{t} \sum_{j=\ell}^{\infty} \overline{i_{j}} t^{j}=\ell \quad \text { if } \overline{i_{\ell}} \neq \overline{0} .
$$

For every $k \in \mathbb{Z}$, the function $t^{k} \mathbb{F}_{p}((t)) \ni c \mapsto t^{\ell-k} c \in t^{\ell} \mathbb{F}_{p}((t))$ is a homeomorphism w.r.t. the topology induced by the $t$-adic valuation. On the other hand, $K=\bigcup_{k \in \mathbb{Z}} t^{k} \mathbb{F}_{p}((t))$. So we see that $\mathbb{F}_{p}((t))$ is the union over an increasing chain of mutually homeomorphic fractal spaces.

However, we wish to show that $\mathbb{F}_{p}((t))$ is locally fractal in a stronger sense. The idea is to write $\mathbb{F}_{p}((t))$ as a union over a collection of mutually homeomorphic fractal subspaces and extend the functions we have used for 
$B_{0}(0)=\mathbb{F}_{p}[[t]]$ in a suitable way so that they work simultaneously for all of these subspaces. To this end, we observe that for any two $a, b \in K$, the function $B_{0}(a) \ni c \mapsto c-a+b \in B_{0}(b)$ is a homeomorphism. Note that for each $\ell<0$ there are only finitely many elements in $B_{\ell}(0)=t^{\ell} \mathbb{F}_{p}[[t]]$ that are non-equivalent modulo $B_{0}(0)$, so we can write $B_{\ell}(0)$ as a finite union of the form $\bigcup_{j} B_{0}\left(a_{j}\right)$.

Example 18. We extend the functions $f_{i}$ we used for $R=\mathbb{F}_{p}[[t]]$ by setting:

$$
f_{i}\left(\sum_{j=\ell}^{\infty} \overline{i_{j}} t^{j}\right):=\sum_{j=\ell}^{-1} \overline{i_{j}} t^{j}+\bar{i}+\sum_{j=1}^{\infty} \overline{i_{j-1}} t^{j}=\sum_{j=\ell}^{-1} \overline{i_{j}} t^{j}+\bar{i}+t \sum_{j=0}^{\infty} \overline{i_{j}} t^{j} .
$$

For every $a=\sum_{j=\ell}^{\infty} s_{j} t^{j} \in \mathbb{F}_{p}((t))$ with $s_{j} \in \mathbb{F}_{p}$, we have that

$$
B_{0}(a)=a+R=\sum_{j=\ell}^{-1} s_{j} t^{j}+R
$$

Hence,

$$
f_{i}\left(B_{0}(a)\right)=f_{i}\left(\sum_{j=\ell}^{-1} s_{j} t^{j}+R\right)=\sum_{j=\ell}^{-1} s_{j} t^{j}+\bar{i}+t R,
$$

and therefore,

$$
\bigcup_{i=0}^{p-1} f_{i}\left(B_{0}(a)\right)=\sum_{j=\ell}^{-1} s_{j} t^{j}+\bigcup_{i=0}^{p-1}(\bar{i}+t R)=\sum_{j=\ell}^{-1} s_{j} t^{j}+R=B_{0}(a) .
$$

Choose any $m \geq 1$ and $i_{0}, \ldots, i_{m-1} \in\{0, \ldots, p-1\}$. By induction on $m$ we derive from (7):

$$
\begin{aligned}
f_{i_{0}} \circ \cdots \circ f_{i_{m-1}}\left(B_{0}(a)\right) & =\sum_{j=\ell}^{-1} s_{j} t^{j}+\overline{i_{0}}+\overline{i_{1}} t+\cdots+\overline{i_{m-1}} t^{m-1}+t^{m} R \\
& =B_{m}\left(\sum_{j=\ell}^{-1} s_{j} t^{j}+\sum_{j=0}^{m-1} \overline{i_{j}} t^{j}\right) \subset B_{0}(a) .
\end{aligned}
$$

For arbitrary discretely valued fields $(K, v)$ with valuation $\operatorname{ring} R$ and valuation ideal $M$, we can proceed as follows. As before, we choose a uniformizing parameter $t \in K$ and a system of representatives $S \subset R$ for the residue field $K v$. We set

$$
K^{-}:=\left\{\sum_{j=\ell}^{-1} s_{j} t^{j} \mid 0 \geq \ell \in \mathbb{Z} \text { and } s_{\ell}, \ldots, s_{-1} \in S\right\} .
$$

Then for every $a \in R$ there is a unique element $a^{-} \in K^{-}$such that $a-a^{-} \in R$. For every $s \in S$ we define a function $f_{s}: K \rightarrow K$ by

$$
f_{s}(a):=a^{-}+s+t\left(a-a^{-}\right) .
$$


For every $a \in K$ we obtain that

$$
f_{s}\left(B_{0}(a)\right)=a^{-}+s+t R
$$

and therefore,

$$
\bigcup_{s \in S} f_{s}\left(B_{0}(a)\right)=a^{-}+\bigcup_{s \in S}(s+t R)=a^{-}+R=B_{0}(a) .
$$

Choose any $m \geq 1$ and $s_{0}, \ldots, s_{m-1} \in S$. By induction on $m$ we derive from (8):

$$
\begin{aligned}
f_{s_{0}} \circ \cdots \circ f_{s_{m-1}}\left(B_{0}(a)\right) & =\sum_{j=\ell}^{-1} a_{j} t^{j}+s_{0}+s_{1} t+\cdots+s_{m-1} t^{m-1}+t^{m} R \\
& =B_{m}\left(a^{-}+\sum_{j=0}^{m-1} s_{j} t^{j}\right) \subset B_{0}(a) .
\end{aligned}
$$

Take $b, c \in B_{0}(a)$. Then $b-c \in B_{0}(0)$, and if $b-c \in B_{m}(0)$ with $m \geq 0$, then $b^{-}=c^{-}$and

$f_{s}(b)-f_{s}(c)=b^{-}+s+t\left(b-b^{-}\right)-\left(c^{-}+s+t\left(c-c^{-}\right)\right)=t(b-c) \in B_{m+1}(0)$.

This shows that each $f_{s}$ is contracting and hence continuous in the topology induced by the ultrametric.

We define:

Definition 19. A locally compact metric space $(X, d)$ is locally fractal if it is the union over a collection of mutually homeomorphic subspaces $X_{j}, j \in J$, and there is a system $F=\left[f_{1}, \ldots, f_{n}\right]$ of functions $f_{i}: X \rightarrow X$ such that for every $j \in J, X_{j}$ is fractal w.r.t. the restrictions of the functions $f_{i}$ to $X_{j}$.

Definition 20. A locally compact topological space $X$ is locally fractal if it is the union over a collection of mutually homeomorphic subspaces $X_{j}, j \in J$, and there is a system $F=\left[f_{1}, \ldots, f_{n}\right]$ of functions $f_{i}: X \rightarrow X$ such that for every $j \in J, X_{j}$ is (topologically) fractal w.r.t. the restrictions of the functions $f_{i}$ to $X_{j}$.

Note that we do not require the functions $f_{i}$ to be continuous or contracting or to satisfy (SC) on all of $X$. Indeed, the functions we constructed above have the property that if $(a-b)^{-} \neq 0$, then $\left(f_{i}(a)-f_{i}(b)\right)^{-}=(a-b)^{-}$.

We have proved:

Proposition 21. Every discretely valued field with finite residue field is locally fractal under both definitions.

Open Access. This article is distributed under the terms of the Creative Commons Attribution 4.0 International License (http://creativecommons.org/licenses/ by/4.0/), which permits unrestricted use, distribution, and reproduction in any medium, provided you give appropriate credit to the original author(s) and the source, provide a link to the Creative Commons license, and indicate if changes were made. 


\section{References}

[1] Banakh, T., Nowak, M.: A 1-dimensional Peano continuum which is not an IFS attractor. Proc. Am. Math. Soc. 141, 931-935 (2013)

[2] Banakh, T., Kubiś, W., Novosad, N., Nowak, M., Strobin, F.: Contractive function systems, their attractors and metrization. Topol. Methods Nonlinear Anal. 46, 1029-1066 (2015)

[3] Banakh, T., Nowak, M., Strobin, F.: Detecting topological and Banach fractals among zero-dimensional spaces. Topol. Appl. 196:A, 22-30 (2015)

[4] D'Aniello, E.: Non-self-similar sets in $[0,1]^{N}$ of arbitrary dimension. J. Math. Anal. Appl. 456, 1123-1128 (2017)

[5] D'Aniello, E., Steele, T.H.: Attractors for iterated function systems. J. Fractal Geom. 3, 95-117 (2016)

[6] Engler, A.J., Prestel, A.: Valued Fields. Springer Monographs in Mathematics. Springer, Berlin (2005)

[7] Nowak, M.: Topological classification of scattered IFS-attractors. Topol. Appl. 160, 1889-1901 (2013)

[8] Ribenboim, P.: Théorie des valuations, 2nd edn. Les Presses de l'Université de Montréal, Montréal (1968)

[9] Ribenboim, P.: The Theory of Classical Valuations. Springer Monographs in Mathematics. Springer, New York (1999)

[10] Warner, S.: Topological Fields. Mathematics Studies, vol. 157. North-Holland, Amsterdam (1989)

\section{JAN DOBROWOLSKI}

Faculty of Mathematics and Physical Sciences,

University of Leeds,

Leeds LS2 9JT,

UK

e-mail: J.Dobrowolski@leeds.ac.uk

Franz-VikTOR KuHLMANN

Institute of Mathematics,

University of Szczecin,

ul. Wielkopolska 15,

70-451, Szczecin,

Poland

e-mail: fvk@math.usask.ca

Received: 18 November 2017 